\title{
Modeling Kinetics of Distortion in Porous Bi-layered Structures
}

Tadesse Molla, Tesfaye; Frandsen, Henrik Lund; Bjørk, Rasmus; Ni, De Wei; Olevsky, Eugene; Pryds, Nini

Published in:

Journal of the European Ceramic Society

Link to article, DOI:

10.1016/j.jeurceramsoc.2012.12.019

Publication date:

2013

Link back to DTU Orbit

Citation (APA):

Tadesse Molla, T., Frandsen, H. L., Bjørk, R., Ni, D. W., Olevsky, E., \& Pryds, N. (2013). Modeling Kinetics of Distortion in Porous Bi-layered Structures. Journal of the European Ceramic Society, 33(7), 1297-1305.

https://doi.org/10.1016/j.jeurceramsoc.2012.12.019

\section{General rights}

Copyright and moral rights for the publications made accessible in the public portal are retained by the authors and/or other copyright owners and it is a condition of accessing publications that users recognise and abide by the legal requirements associated with these rights.

- Users may download and print one copy of any publication from the public portal for the purpose of private study or research.

- You may not further distribute the material or use it for any profit-making activity or commercial gain

- You may freely distribute the URL identifying the publication in the public portal 


\title{
Modeling Kinetics of Distortion in Porous Bi-layered Structures
}

\author{
Tesfaye Tadesse Molla ${ }^{1 *}$, Henrik Lund Frandsen ${ }^{1}$, Rasmus Bjørk ${ }^{1}$, De Wei Ni ${ }^{1}$, Eugene Olevsky ${ }^{2}$, Nini \\ Pryds $^{1}$ \\ ${ }^{1}$ Technical University of Denmark, Department of Energy Conversion and Storage, Ris $\varnothing$ Campus \\ Frederiksborgvej 399, P.O. Box 49, Building 779, 4000 Roskilde, Denmark \\ ${ }^{2}$ San Diego State University, Mechanical Engineering Department, 5500 Campanile Dr., San Diego, CA \\ 92182-1323, USA
}

\begin{abstract}
Shape distortions during constrained sintering experiment of bi-layer porous and dense cerium gadolinium oxide (CGO) structures have been modeled. Technologies like solid oxide fuel cells require co-firing thin layers with different green densities, which often exhibit differential shrinkage because of different sintering rates of the materials resulting in undesired distortions of the component. An analytical model based on the continuum theory of sintering has been developed to describe the kinetics of densification and distortion in the sintering processes. A new approach is used to extract the material parameters controlling shape distortion through optimizing the model to experimental data of free shrinkage strains. The significant influence of weight of the sample (gravity) on the kinetics of distortion is taken in to consideration. The modeling predictions indicate good agreement with the results of sintering of a bi-layered CGO system in terms of evolutions of bow, porosities and also layer thickness.
\end{abstract}

Keywords: Modeling, Sintering, Bi-layer, Distortion

*Corresponding author:- Address: Frederiksborgvej 399, P.O. Box 49, Building 779, 4000 Roskilde, Denmark, Tele: +45 2074

5931, Fax: +45 4677 5858, e-mail: ttmo@dtu.dk 


\section{Introduction}

Discretely graded ceramic multi-layers are considered to be promising material structures due to their performances in the development of various energy efficient electromechanical systems ${ }^{(1,2)}$. These structures are often produced by laminating different porous layers and then sintering them together (cofiring). During co-firing of multi-layers, different densification rates can cause development of stresses leading to defects like cracks and macrostructural distortions ${ }^{(3-11)}$. Asymmetric arrangement of layers usually relaxes the mismatched stress evolutions by warping and hence creating instabilities in the shape of the component. For example in the case of planar solid oxide fuel cell (SOFC) technologies, the deformations in the shape of the components/cells reduce successful stack assembly, and thus it is not desired. Therefore there is a growing interest for understanding how the intrinsic material properties can affect the evolution of distortion in order to reduce the stress development and to allow components to be produced with the desired shape after co-firing. In this study, this is studied through a combination of sintering experiments and mechanical modeling.

The introduction of continuum mechanics with linear viscous material model for porous structures can be seen as an important development in addressing the problem of shape distortions during co-firing of ceramic layers ${ }^{(12,13)}$. Since then there have been a number of reported works that deal with distortions in bi-layer ceramic systems. One of these is the work by Lu et al. ${ }^{(14)}$, in which the continuum model of sintering is used to describe the kinetics of densification and curvature evolution, taking the effect of particle coarsening and grain growth into consideration. Lu et al. also considered the impact of pore size on the densification behavior of each layer into account. From beam theory, the stress and strain distributions along the section of the layers are known to be linear, but in the model by Lu et al. a uniform strain distributions are assumed over each layer, which may affect the accuracy of the model.

Detailed work on experimental observation of processing defects and the corresponding viscoelastic stress computation for constrained densification of Alumina/Zirconia hybrid laminates has been published by Cai et $a l .{ }^{(3,15)}$ After measuring the viscous properties of the constituent layers using cyclic loading 
dilatometry, Cai et al. were able to model the bow evolution of bi-layers in good agreement with experimental results. Cai et al. however did not consider the evolution of thickness of each layer during densification, which is significant in the case of highly porous layers ${ }^{(8,16)}$.

The linear distribution of strains with the corresponding evolution of thicknesses in each layer has been considered in the model suggested by Kanters et al. ${ }^{(1)}$. Kanters et al. reported a good agreement of the model prediction of curvature evolution for two types of bi-layer samples made from nanocrystalline yttria-stabilized zirconia with different thicknesses.

Kang et al. ${ }^{(4)}$ used the models proposed by Cai et al. and Kanters et al. to study a bi-layered system of Gadolinium-doped ceria and a cermet of Nickel oxide in a backbone structure of Yttria stabilized zirconia. In both cases, they found a good agreement of distortion evolutions with the measurements during the sintering. In a similar way, Ollagnier et al ${ }^{(8)}$ compared the models of Cai et al. and Kanters et al. on bi-layers of porous and dense low-temperature co-fired ceramics (LTCC) with different initial thickness ratios. Unlike Kang et al., they found a significant discrepancy between the model predictions and the measurements of camber for which anisotropy of sintering parameters, effects of gravity and heating rates were suggested as a cause. Ollagnier et al. also showed the influence of ratio of initial thicknesses of the bi-layered system on the amount of camber after the sintering. The importance of gravity is also suggested by Mücke et $a .^{(2)}$ after their experimental observations on SOFC samples prepared from 8YSZ. Mücke et al. compared curvature evolutions of two samples sintered in vertical and horizontal positions. They observed reduction in the camber development in the case of horizontally sintered sample in which the effect of gravity is significant ${ }^{(2)}$.

Modeling the mismatch stresses and curvature of bi-layered structures with the help of experimental characterization of the viscous properties of each layer using cyclic load dilatometry has also been reported by Chiang et al. ${ }^{(5)}$ and Ravi and Green ${ }^{(9)}$. 
Often experimental characterizations of the viscous behavior of each layer are used to model the curvature evolution during the sintering process. This requires another set of creep experiments to independently measure the viscous behaviors of individual layers ${ }^{(3-10)}$. In most of the works reported, techniques like cyclic loading ${ }^{(5,9)}$ and sinter forging ${ }^{(4)}$ are usually used. Cologna et al. ${ }^{(17)}$ also proposed another technique, called vertical sintering, in which the sample is allowed to sinter vertically under the influence of its own gravity. A similar way of determining the viscosity of each layer by measuring the maximum deflection rate for beams of porous materials that are allowed to deform under their own weight or under applied loads was also suggested by Atkinson et al and Lee et al. ${ }^{(18,19)}$. Alternative to these experiments, the capabilities of proven modeling approaches, like the Skorohod Olevsky Viscous Sintering (SOVS) model ${ }^{(12,13)}$, could also be used together with one sintering experiment conducted simultaneously for individual layers and asymmetric bi-layer so as to study the kinetics of densification in the free layers and shape distortions in the bi-layer system.

The effect of differential shrinkage is explained very well to be the factor controlling distortion. But as the studies by Mücke et al. and Ollagnier et al. showed the weight of the sample (gravity) also affects the rate of distortion by being an additional factor generating creep in the porous layers. Thus with all the important contributions from the works cited, it is still necessary to modify the modeling approaches so as to improve the accuracy of the predictions while maintaining them simple. The work by Frandsen et al. ${ }^{(16)}$, from which the basis for the modeling approach adopted in this study, is built on a viscous analogy of classical laminate theory, where the effect of weight of the sample (gravity) on the distortion is considered.

The objective of this study is to present an alternative way of obtaining material parameters that control shape distortion from a single dilatometry experiment so as to model kinetics of densification and distortion in the bi-layer system. Improved modeling approaches are used in such a way that the effect of weight of the sample on the distortion evolution is considered to be another stress generating factor in addition to the differential shrinkage. Also the thickness evolutions in each layer are considered through 
the effective densification of each layer in the thickness directions. The approach is applied to obtain the kinetics of shrinkage and bow development during the sintering of porous and dense cerium gadolinium oxide, $\mathrm{Ce}_{0.9} \mathrm{Gd}_{0.1} \mathrm{O}_{1.95-\mathrm{d}}$, (CGO) layers with the help of analytical methods implemented in Matlab.

\section{Cosintering Model}

The analysis is made based on continuum theory of sintering, which describes the macrostructural behavior of a porous body during sintering. It relates the external load to the strain rate by nonlinear viscous constitutive relationship ${ }^{(12,13)}$. The continuum model for linear relationship between the equivalent stress and strain rates is given by:

$$
\sigma_{i j}=2 \eta_{0}\left[\varphi \dot{\varepsilon}_{i j}+\left(\psi-\frac{1}{3} \varphi\right) \dot{e} \delta_{i j}\right]+P_{L} \delta_{i j}
$$

where $\eta_{0}$ is the shear viscosity of the fully dense materials, $\varphi$ and $\psi$ are the normalized shear and bulk viscosities, $P_{L}$ is the effective driving potential for sintering or sintering stress, $\delta_{i j}$ the Kronecker delta and $\dot{\varepsilon}_{i j}$ and $\dot{e}$ are the total and bulk strain rates respectively related to the stress tensor $\sigma_{i j}{ }^{(12,13)}$. The normalized shear and bulk viscosities are considered to be functions of porosity or volume fractions of voids in the porous body, $\theta$, see Eq. (2). The effective sintering stress is the product of normalized sintering stress and local sintering stress, which is a function of surface energy per unit area, $\alpha$, and grain size, $G$, in the form shown by Eq. (3).

$$
\begin{gathered}
\varphi=(1-\theta)^{2} ; \psi=\frac{2}{3} \frac{(1-\theta)^{3}}{\theta} \\
P_{L}=\frac{3 \alpha}{2 G}(1-\theta)^{2}
\end{gathered}
$$

The porosity evolution is related to the volumetric densification strain using the principle of mass conservation as ${ }^{(12)}$ : 


$$
\dot{e}=\frac{\dot{\theta}}{1-\theta}
$$

The axial shrinkage rate for freely sintering sample can be found from the general model in Eq. (1) using the effective sintering stress, $P_{L}$ as:

$$
\dot{\varepsilon}^{f}=-\frac{P_{L}}{6 \eta_{0} \psi}
$$

The relationship between the stress tensor, $\sigma_{i j}$, and the viscous (creep-related) strain rate tensor, $\dot{\varepsilon}_{i j}^{\prime}$, can be deduced from Eq. (1) as ${ }^{(12)}$ :

$$
\sigma_{i j}=2 \eta_{0}\left[\varphi \dot{\varepsilon}_{i j}^{\prime}+\left(\psi-\frac{1}{3} \varphi\right) \dot{e}^{\prime} \delta_{i j}\right]
$$

For a detailed description of the continuum theory of sintering, especially of its microstructural assumptions, please refer the work by Olevsky ${ }^{(12)}$. Note that possible anisotropies of the sintering parameters and of the pore-grain structure are not included in this analysis.

The shear viscosities of the fully dense bodies are assumed to vary with temperature, $T$, in each layer according to the Arrhenius type of viscosity function, see Eq. (7), as suggested by Reiterer et al. ${ }^{(20)}$ to be suitable for the SOVS model.

$$
\eta_{0}=A T \exp \left(\frac{Q_{s}}{R T}\right)
$$

Here $A$ is the pre-exponential constant, $Q_{s}$ the apparent activation energy for the range of density in consideration irrespective of the specific transport mechanism associated with the material and $R$ is the universal gas constant.

The simultaneous grain growth during the sintering process can be considered using a function of time, $t$, limited by the activation energy for grain growth, $Q_{g}$, as shown in Eq. (8) ${ }^{(21,28)}$. Here $n$ is the grain growth exponent depending on the creep or diffusion mechanism (e.g. $n=2$ for Nabarro-Herring creep and $n=3$ for Coble creep) and $k_{o}$ is the pre-exponential constant ${ }^{(21)}$. 


$$
G^{n}=G_{0}^{n}+k_{0} \exp \left(\frac{-Q_{g}}{R T}\right) t
$$

\section{(1)Stresses in bi-layer system}

Consider a bi-layer geometry consisting of thin dense layer over thick porous layer. The stresses developed in the bi-layer system that lead to bending of the sample are assumed to be because of two phenomena occurring simultaneously during co-firing. These are 1) creep due to stresses from differential shrinkage and 2) creep induced by the sample own weight (gravity).

As shown by the schematics of a sectioned bi-layer system in Figure 1a, the relative difference in the rate of shrinkage between a porous thick layer and dense thin layer creates the densification mismatch, which leads to an internal in-plane force, $N^{f}$, and the bending moment, $M^{f}$. For the sample geometry the stress normal to the interface is very small compared to the in-plane stresses $\left(\sigma_{z}=0\right)$. So the relative difference in shrinkage generates a biaxial state of sintering stress $\left(\sigma=\sigma_{x}=\sigma_{y}\right)$ and a bending moment that bends the sample towards the porous layer. Considering the above assumptions in Eq. (6), the viscous or creep strain rates, $\dot{\varepsilon}^{\prime}$, can be described using the biaxial stress:

$$
\begin{gathered}
\dot{\varepsilon}^{\prime}=\dot{\varepsilon}_{x}^{\prime}=\dot{\varepsilon}_{y}^{\prime}=\frac{3 \psi+2 \varphi}{18 \eta_{0} \varphi \psi} \sigma ; \dot{\varepsilon}_{z}^{\prime}=-\frac{6 \psi-2 \varphi}{18 \eta_{0} \varphi \psi} \sigma \\
\sigma=E_{b}^{\prime} \dot{\varepsilon}^{\prime}=>E_{b}^{\prime}=\eta_{0} \frac{18 \varphi \psi}{3 \psi+2 \varphi}
\end{gathered}
$$

This phenomenon can be considered as a creep process due to the internal sintering stress where the biaxial stress is linearly related to the viscosity of the porous body, $E_{b}^{\prime}$, which is the product of viscosity of the fully dense material and a time dependent function of porosity, see Eq. (10) ${ }^{(16)}$. Note that in this work, positive curvature is defined when the sample bows towards the porous layer. 


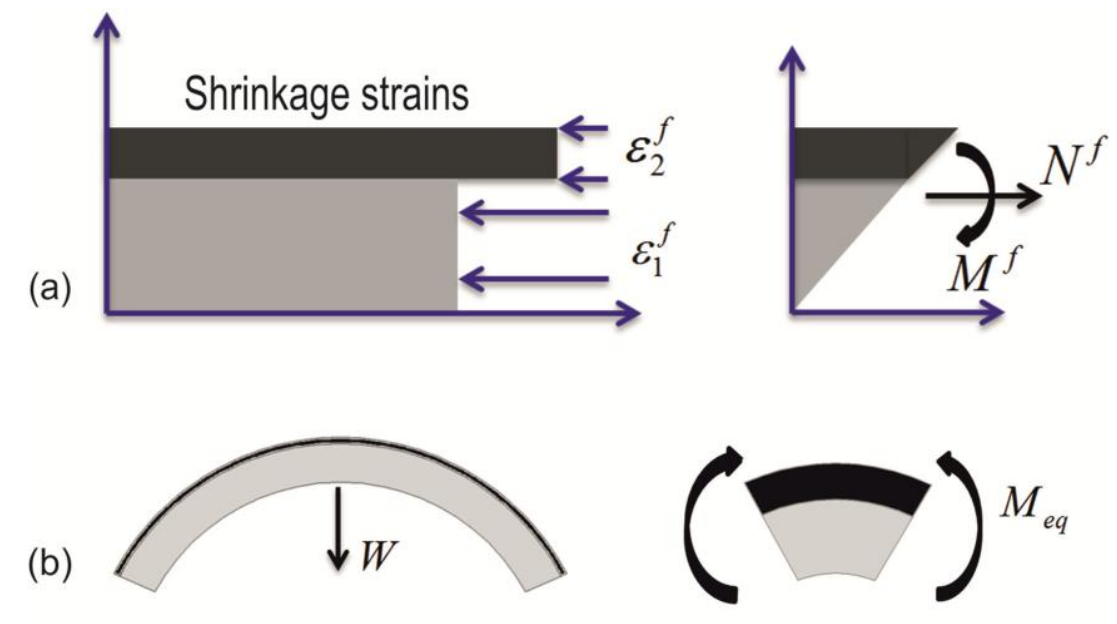

Figure 1: Schematics showing stress in sintering of bi-layer structure (a) due to relative difference in shrinkage \& (b) due to creep induced by sample own weight.

Creep induced by the sample own weight, $W$, while it sinters is also another phenomena generating stresses in the bi-layer structure. Unlike creep due to the internal sintering stress, which deforms the structure towards the porous layer, creep due to the weight of the body will oppose the deformation (e.g. negative curvature) of a flat sintering tape. The reason for this is explained schematically in Figure $1 \mathrm{~b}$. Since the width of the sample is small compared to its length, the structure can be considered as a beam with evenly distributed weight over its length. Therefore, the creep generates only a uniaxial state of stress $\left(\sigma=\sigma_{x}\right.$ ) due to the equivalent bending moment, $M_{e q}$, which opposes the bending due to mismatch of the sample. Consideration of these assumptions into Eq. (6) gives the corresponding viscous or creep strain rates as:

$$
\begin{gathered}
\dot{\varepsilon}^{\prime}=\dot{\varepsilon}_{x}^{\prime}=\frac{6 \psi+\varphi}{18 \eta_{0} \varphi \psi} \sigma ; \quad \dot{\varepsilon}_{y}^{\prime}=\dot{\varepsilon}_{z}^{\prime}=-\frac{3 \psi-\varphi}{18 \eta_{0} \varphi \psi} \sigma \\
\sigma=E_{u}^{\prime} \dot{\varepsilon}^{\prime}=>E_{u}^{\prime}=\eta_{0} \frac{18 \varphi \psi}{6 \psi+\varphi}
\end{gathered}
$$


Here also the uniaxial stress is considered to be linearly proportional to other viscosity of the porous body, $E_{u}^{\prime}$, which is again the product of viscosity of the fully dense material and another function of porosity, see Eq. (12) ${ }^{(16)}$.

\section{(2)Kinetics of shrinkage and distortion in the bi-layer}

In the present work, linear distribution of the constrained strain rate across the section of each layer is assumed. This will represent the reality better than the uniform strain rate. The viscous analogy of classical laminate theory has been used with the evolving curvature rate, $\dot{\kappa}$, and longitudinal strain rate, $\dot{\varepsilon}_{0}$, to describe the linear strain rate distribution, $\dot{\varepsilon}$, across the thickness. This is shown in Eq. (13) with $z$ representing the vertical coordinate of a point in the layer.

$$
\dot{\varepsilon}=\dot{\varepsilon}_{0}+\dot{\kappa} z
$$

The corresponding distribution of stress is also linear with the generalized material viscosity, $E^{\prime}$, which depends on the evolving viscosity of the porous body, see Eqs. (10) and (12), and the difference between the constrained, $\dot{\varepsilon}$, and free or unconstrained shrinkage rates, $\dot{\varepsilon}^{f(16)}$.

$$
\begin{gathered}
\sigma=E^{\prime} \dot{\varepsilon}^{\prime}=E^{\prime}\left(\dot{\varepsilon}-\dot{\varepsilon}^{f}\right)=E^{\prime}\left(\dot{\varepsilon}_{0}+\dot{\kappa} z-\dot{\varepsilon}^{f}\right) \\
E^{\prime}= \begin{cases}E_{b}^{\prime} & \text { for biaxial stress } \\
E_{u}^{\prime} & \text { for uniaxial stress }\end{cases}
\end{gathered}
$$

The free shrinkage rates are calculated using Eq. (5) and the longitudinal strain rates, $\dot{\varepsilon}_{0}$, and the curvature rate, $\dot{\kappa}$, can be evaluated applying the equilibrium condition with the viscous analogy of classical laminate theory corresponding to the stresses and the bending moments in both layers as ${ }^{(16)}$ :

$$
\begin{aligned}
& \int_{\text {both layers }} \sigma d z=0 \\
& \int_{\text {both layers }} \sigma z d z=0
\end{aligned}
$$


Therefore the constrained strain rate, $\dot{\varepsilon}$, can be obtained with the help of Eq. (13) for the coordinate point defined by $z$. The evolving porosity, $\theta$, and the thickness, $h$, of each layer in the bi-layer are also updated through time considering the total stress states in each layer during the co-firing.

The lateral contraction ratios, $v^{\prime}$, can be derived considering the viscous strains for each of the layers as shown in Eq. (9) and (11). These parameters are related to porosity using the normalized shear and bulk viscosities for each stress conditions as shown by Eq. (18).

$$
v^{\prime}=-\frac{\dot{\varepsilon}_{z}^{\prime}}{\dot{\varepsilon}_{x}^{\prime}}= \begin{cases}\frac{6 \psi-2 \varphi}{3 \psi+2 \varphi} & \text { for biaxial stress } \\ \frac{3 \psi-\varphi}{6 \psi+\varphi} & \text { for uniaxial stress }\end{cases}
$$

Thus in the SOVS model the lateral contraction ratios are independent of the material and only depend on the porosity of the body. This is an assumption built into the SOVS framework, and in general these parameters are difficult to measure as the lateral strain rate is small compared to other strain rate components, i.e. the longitudinal strain rate and sintering strain rates.

The above approaches can be used to solve two parallel problems simultaneously accounting for the biaxial stresses due to differential shrinkage and the uniaxial stresses induced by sample's own weight (gravity). The curvature evolution due to the weight of the sample could be approximated by assuming a constant equivalent bending moment, $M_{e q}{ }^{(16)}$. The total curvature rate is then calculated simulating the simultaneous effects in the same co-ordinate system. Further theoretical details and derivations of the model described above can be found in Frandsen et al. ${ }^{(16)}$.

All the information for shrinkages and curvature development can be integrated through time according to the sintering profile used in the experiment if the viscosities of both layers at fully dense state, i.e. $\eta_{01}$ and $\eta_{02}$, and the grain growth kinetics in each layer, $G_{1}$ and $G_{2}$, are known. 


\section{(3)Obtaining the constitutive parameters}

In this work, an alternative approach to experimentations has been employed to find the viscous material parameters required for modeling the shrinkage and curvature development observed during the entire sintering process. This is achieved through first modeling the free shrinkage behaviors of individual layers during the sintering process. The free densification strain rate, $\dot{\varepsilon}^{f}$, can be described explicitly by combining Eqs. (2), (3) and (5) with the fully dense body viscosity given by Eq. (7).

$$
\dot{\varepsilon}^{f}=-\frac{3}{8} \frac{\alpha}{A G T} \exp \left(\frac{-Q_{s}}{R T}\right)\left(\frac{\theta}{1-\theta}\right)
$$

The surface energy per unit area in each layer can be estimated which is often in order of $1 \mathrm{~J} / \mathrm{m}^{2}$ for most ceramic oxides ${ }^{(1,28)}$. Consider a co-firing process with a temperature profile that constitutes an iso-rate followed by isothermal stages. The grain sizes at the onset of the iso-rate sintering, i.e. $G_{01}$ and $G_{02}$, and those at the onset of isothermal temperature, e.g. $G_{1}^{\prime}$ and $G_{2}^{\prime}$, could be used to estimate the grain growth pre-exponential factors, $k_{01}$ and $k_{02}$, see Eq. (8), if the activation energies for grain growth are known. This would help to approximate the grain growth kinetics in the two layers during the entire sintering process as per the sintering temperature profile.

The model predictions for free shrinkage strain rate, $\dot{\varepsilon}^{f}$, in each layer can be optimized with the respective experimental data. This can be done by starting the model simulations with realistic guesses of the four unknown parameters of the two layers, i.e. $A_{1}, A_{2}$ and $Q_{s 1}, Q_{s 2}$. The unknown parameters can then be identified as those providing the minimum deviation as per Eq. (20) between the experimental data and model simulation.

$$
\Delta=\left\{\sum_{i=1}^{N}\left[\left(\frac{\varepsilon_{1}^{f, \operatorname{Sim}}-\varepsilon_{1}^{f, \operatorname{Exp}}}{\operatorname{mean}\left(\varepsilon_{1}^{f, \operatorname{Exp}}\right)}\right)^{2}+\left(\frac{\varepsilon_{2}^{f, \operatorname{Sim}}-\varepsilon_{2}^{f, \operatorname{Exp}}}{\operatorname{mean}\left(\varepsilon_{2}^{f, \operatorname{Exp}}\right)}\right)^{2}\right]\right\}^{\frac{1}{2}}
$$


where $\varepsilon^{f}$ is free shrinkage strain in the individual layers and the sum is taken over all $N$ data points in time. A similar methodology has been used by Garino and Bowen ${ }^{(22)}$ to estimate the constrained shrinkage of glass powders using free shrinkage measurement data and the viscous flow sintering model suggested by Scherer ${ }^{(23)}$. Therefore the approach adopted here could be used to approximate the temperature dependent viscous behaviors of fully dense bodies as per the Arrhenius type of viscosity function given in Eq. (7) without the need for other creep experiments.

\section{Model verification and Discussion}

In this study, the experimental data reported by De et al. ${ }^{(24)}$ on tapes made from CGO (Rhodia S.A., France) with a specific surface area of $6.6 \mathrm{~m}^{2} / \mathrm{g}(\mathrm{d} 50$ particle size $0.2 \mu \mathrm{m})$ are used for validating the presented modeling approach. De et al. fabricated samples that consisted of two layers of CGO tapecasted on top of each other. One of the layers contained a significant amount of pre-calcined powder and graphite powder (V-UF1 99.9, Graphit Kropfmühl, Germany) as a pore former and will be referred to as CGO_P. The other layer has a higher relative density and will be referred to as CGO_D. At the beginning of the sintering, the relative density and initial thickness of the porous layer was 23 vol\% and $380 \mu \mathrm{m}$ respectively. The corresponding dense layer was 56 vol\% dense and $27 \mu \mathrm{m}$ thick initially. The dense and porous tapes of length $24.5 \mathrm{~mm}$ and width $5.1 \mathrm{~mm}$ were laminated. The samples were subsequently cofired in a furnace with a temperature-time profile, which consists of iso-rate firing with $0.83^{\circ} \mathrm{C} / \mathrm{min}$ from $400^{\circ} \mathrm{C}$ to $1100^{\circ} \mathrm{C}$ followed by an isothermal sintering at $1100^{\circ} \mathrm{C}$ for 4 hours. Individual samples of each layer were also sintered in the same furnace in order to observe the free shrinkage of each layer. The evolutions of curvature in the bi-layer sample and shrinkage of the free samples were recorded in-situ using a high temperature furnace equipped with an optical dilatometer (Fraunhofer-Institute Silicateforschung, Germany). SEM micrographs taken by De et al. are also used in this study.

From the sintering experiment considered in this study, the optimization procedure is applied on the two freely sintered samples to estimate the viscous behaviors of the layers based on the pre-exponential 
factors, i.e $A_{1}$ and $A_{2}$, and the apparent activation energies, $Q_{s 1}$ and $Q_{s 2}$. The free shrinkage strains as a function of time for CGO_P and CGO_D layers are shown in Figure 2. The porous layer, CGO_P shows a faster and larger densification (close to 40\%) than the dense layer, CGO_D, which shows less than 20\% of shrinkage.

Based on the optimization procedure applied on the free samples, Figure 2 again shows comparison between results of the model and experimental data for free shrinkage strains. Shrinkage in the dense layer is also observed to bypass the shrinkage in the porous layer for a short time range in the sintering process. The model results are in good agreement with experimental free sintering strain data both for the CGO_P and CGO_D layers.

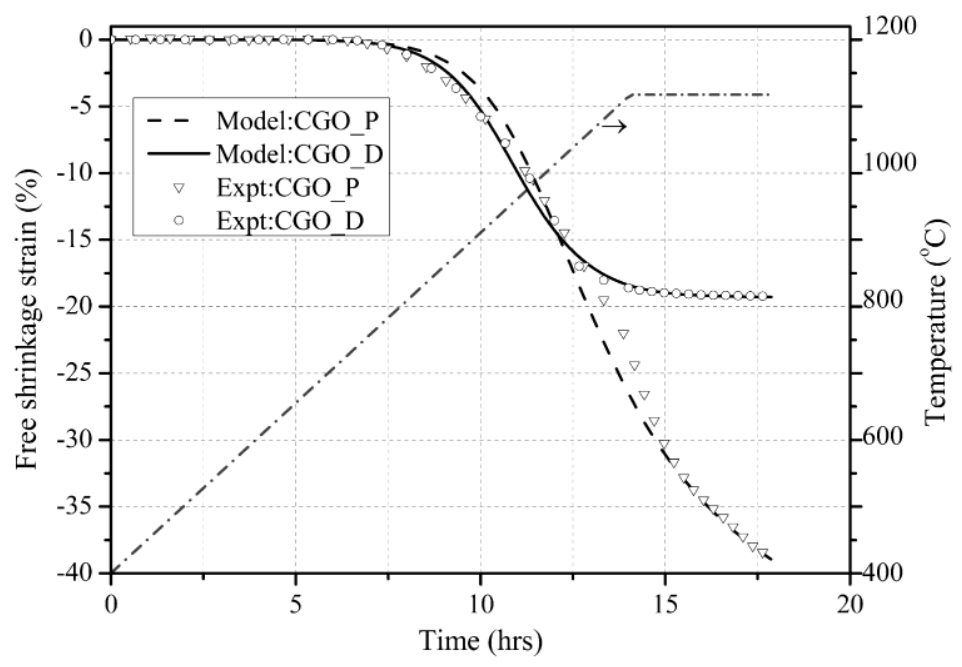

Figure 2: Comparison of the model to the experimental data for free shrinkage strains

The parameters defining the viscous behaviors of each layer extracted from the optimization approach followed in this study are shown in Table 1 . Theoretically, the porous as well as dense layers should have equal values of apparent activation energy, as they are same materials (CGO). But practically there might be differences due to the fact that both layers were prepared with different additions. The other reason for higher apparent activation energy in the case of CGO_D can be due to larger contribution of coarsening to 
microstructural changes at higher sintered densities ${ }^{(21)}$. The influence of grain growth on the viscosity of the fully dense body is not explicitly considered in this work instead both parameters, $A$ and $Q_{s}$, are made free in the fitting for each layer. This is made to lump the influence of grain growth into the viscosity function, $\eta_{0}(T)$. The above assumption of the viscosity function is also consistent with the work reported by Reiterer et al. Note that no attempt has been made to link the $Q_{s}$ in Table 1 to a specific material diffusion mechanism in CGO as suggested by Reiterer et al. Instead they are referred to be the apparent activation energy for the entire densification ranges observed in each layer.

Table 1: Parameters defining the viscous behaviors of the layers

\begin{tabular}{l|c|c}
\hline & $A$ (Pa.s) & $Q_{s}(\mathrm{~kJ} / \mathrm{mol})$ \\
\hline CGO_P & $0.71 \pm 0.153$ & $196 \pm 4$ \\
\hline CGO_D & $0.03 \pm 0.005$ & $208 \pm 3$ \\
\hline
\end{tabular}

The evolutions of uniaxial viscosities of the porous CGO_P and CGO_D as a function of temperature estimated after the respective viscous parameters were found are shown in the Figure 3. The porous layer is shown to have lower uniaxial viscosity compared to the dense one at low temperatures and evolves to be higher than the dense layer at higher temperatures. This is consistent with the high rate of densification observed in the porous layer towards higher temperatures. In addition, the trend in the evolution of the viscosities are also consistent with the results reported by Reiterer et al. for $\mathrm{ZnO}$ showing the influence of temperature at the beginning and the evolving density at higher temperatures. Note that although the two layers are prepared from the same material (CGO), it is believed that the different additives to the CGO_P affected the pore-grain interaction and hence it's viscous behavior.

The contraction ratios are calculated using the porosity evolutions of each layer in the bilayer system and are shown in Figure 4 for uniaxial as well as biaxial stress conditions. Note that the contraction ratio in the biaxial stress condition has nothing to do with Poisson's ratio as the later is defined for particular case 
in viscous problems i.e. for uniaxial relaxation experiments ${ }^{(25)}$. The CGO_P layer is very porous in the beginning of the sintering, and the lateral contraction ratios in Eq. 18 attain unrealistic low values. Therefore minimum value of 0.1 has been imposed. This has no significant influence on the results.

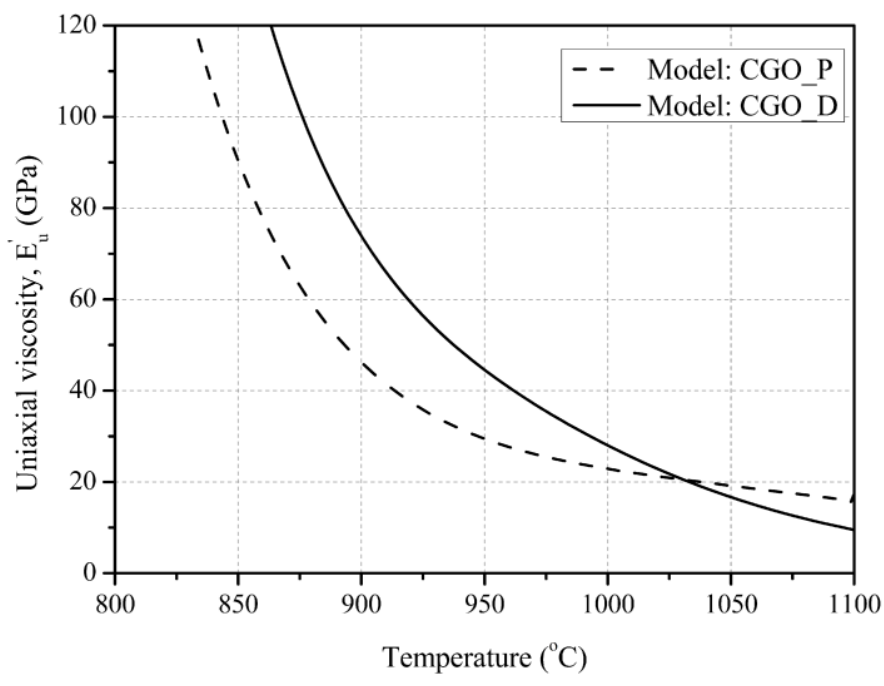

Figure 3: Evolution of viscosity of the porous body of each layer, $E_{u}^{\prime}$, during the iso-rate sintering

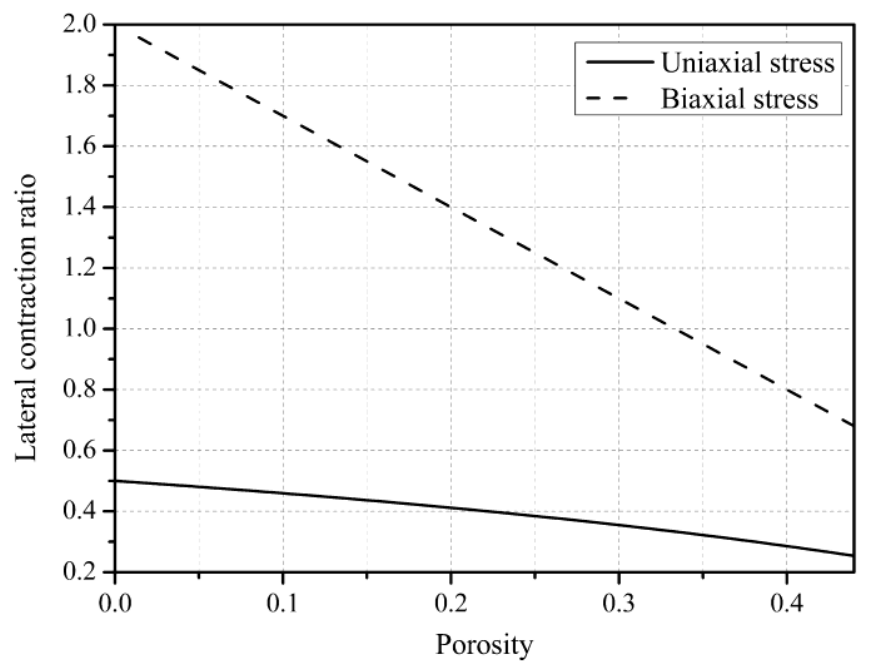

Figure 4: Evolution of the lateral contraction ratios as a function of porosity 
As mentioned earlier, creep experiments of single layered specimens could also have been used to obtain these material parameters. This approach would lead to greater certainty on the viscosity of the individual layer. It would however also require more extensive experimental work, as initial sintering of each layer must be done separately before performing the creep experiments. In a continuous development of new ceramic multi-layers the approach used here might be advantageous due to its simplicity.

While modeling the kinetics of shrinkage and distortion in the bi-layer system, the linear strain distributions across each layer are implemented through the viscous analogy of classical laminate theory which requires a perfect bonding between the bi-layers. This is confirmed by the SEM image observations of a section of the bi-layer at the end of isothermal sintering, see Figure 5a. There were no sintering defects such as cracks or de-lamination in the interface of the CGO_P and CGO_D layers and this was found to be consistent with the studies by J. Jean et al. ${ }^{(26)}$. Cross sectional images of the dense and porous layers at $875^{\circ} \mathrm{C}$ after 2 hours of holding followed by quenching are also shown in Figure $5 \mathrm{~b}$ and c. The difference in the porosity can clearly be seen. 

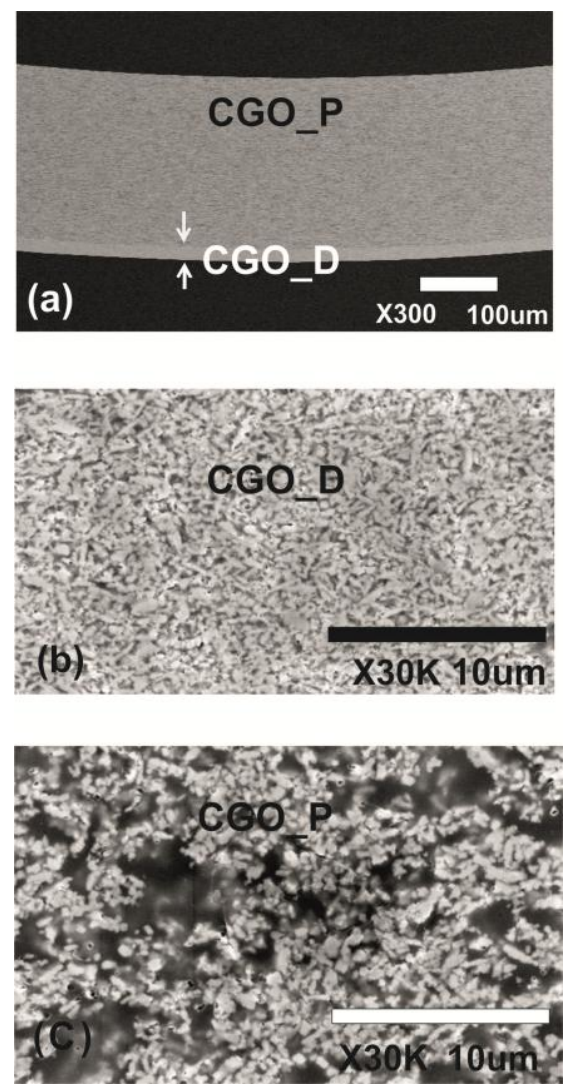

Figure 5: Cross sectional SEM images of the microstructures (a) CGO_D and CGO_P with no defects, (b) CGO_D at $875^{\circ} \mathrm{C}$ after $2 \mathrm{hrs}$ and (c) CGO_P at $875^{\circ} \mathrm{C}$ after $2 \mathrm{hrs}$.

Using the estimated evolutions of viscosity and grain size throughout the entire (iso - rate and isothermal) sintering, densifications and the distortion development in the bi-layer system are modeled for the prescribed temperature profile used in the experiment. The initial porosities in the bi-layer at the onset of the sintering process are assumed to be comparable to the free samples as there were no prior sintering activities. Table 2 shows the different parameters used in the modeling of the entire sintering process. 
Table 2: Material parameters used to model densification and distortion in the bi-layer system

\begin{tabular}{l|c|c|c}
\hline \multicolumn{1}{c|}{ Parameter } & CGO_P & CGO_D & Source \\
\hline Surface energy per unit area $\left(\mathrm{J}^{\prime} \mathrm{m}^{2}\right)$ & 1 & 1 & Estimated \\
\hline Activation energy for grain growth $(\mathrm{kJ} / \mathrm{mol})$ & 413 & 430 & {$[27]$} \\
\hline Initial mean grain size, $G_{0}(\mu \mathrm{m})$ & 0.3 & 0.25 & {$[24]$} \\
\hline Mean grain size at the holding temperature $(\mu \mathrm{m})$ & 1.0 & 1.25 & Estimated \\
\hline Initial mean porosity level $(\%)$ & 77 & 44 & {$[24]$} \\
\hline Initial thickness $(\mu \mathrm{m})$ & 380 & 27 & {$[24]$} \\
\hline Grain growth pre-exponential factors $\left(\mathrm{m}^{3} / \mathrm{s}\right)$ & $1.0 \times 10^{-7}$ & $1.1 \times 10^{-6}$ & Fitted \\
\hline
\end{tabular}

The experimental measurements of shape distortion of the CGO_P/CGO_D bi-layer is shown in Figure 6 in terms of bow, $u$, which is related to curvature, $\kappa$, through the length of the sample. It is observed that initially the bow bends towards the dense layer before turning to the porous layer. The effect of gravity is also observed to be significant during the last hours of sintering mainly in the isothermal regime where a decrease in the bow is clearly observed. This observation is also consistent with those seen by Ollagnier $e t$ $a l .^{(8)}$.

The free sintering samples, especially the porous layer, are however shrinking throughout the experiment as seen on Figure 2. Thus, if not for the gravity, the bow should increase throughout the experiment as well. The reason for the reduction of the bow in the final hours is that the sintering rate of the layers decreases, see Figure 2, but the effect of gravity remains constant. Thus, at the same point the bow rate due to sintering decreases to be below the oppositely directed bow rate of gravity and hence the overall bow decreases.

The model prediction for the distortion evolution shows a good agreement with experimental observation as shown in Figure 6. The model captured the development of the bow towards the dense layer due to the initial faster shrinkage observed in the CGO_D before it is reversed to the porous layer. The availability of pre-calcined powders in the porous layer can make the average initial grain size in the CGO_P larger 
than CGO_D slowing densification in the porous layer initially. This is followed by an initial curvature increase towards the dense layer. Shortly after, however, the high amount of porosity and slower grain growth in the porous layer allows faster densification in the CGO_P followed by curvature increase towards the porous layer.

The model is also able to indicate the decreasing trend of the evolution of bow in the final hours of the sintering due to gravity. For the sake of comparison, the modeling approach reported by Cai et al. is also implemented parallel to the approach followed in this study, see Figure 6. It is clear to see the significance of considering weight of the sample or gravity especially in the last hours of the sintering experiment which is mainly in the isothermal regime. The influence of gravity in the iso-rate sintering is very small as the comparison reflects a similar evolution of bow due to higher sintering activities in both layers. The observed discrepancies between the model and experimental results in Figure 6 are believed to be caused by friction between the sample support surface and of the sample edges while it deforms. Possible anisotropies of densifications from the tape casting might also be an explanation.

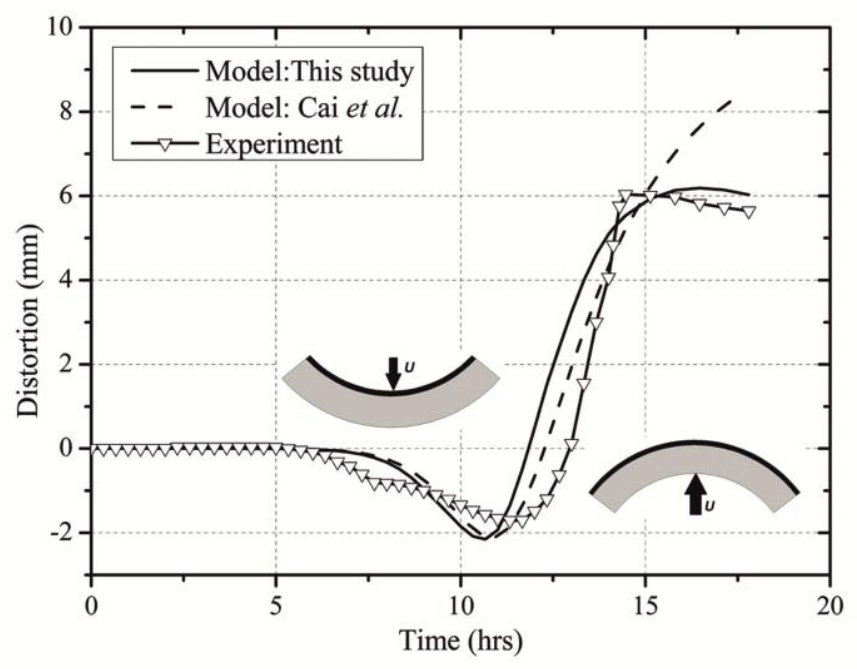

Figure 6: Comparison of the model to the experimental data for distortion $(u)$ during the entire sintering 
Apart from predicting the shape distortion, the model predictions of the final porosities are also in good agreement with the results measured from the SEM studies, see Figure 7. As is expected, the porosity of the CGO_P layer shows a fast and large reduction compared with the reduction of the dense layer, CGO_D. Note that the model predicts only the average value as a function of time in each layer as the porosity variation across the thickness of the layer is beyond the scope of this study. The impact of constraint stress on the evolution of porosity in each layer has also been studied. Figure 8 shows the ratio of porosity evolutions in the constrained and free samples as a function of time. The effect of constraint related stress on the CGO_P is shown to be minor and the porosity evolution in the CGO_P is almost unaffected. This is due to the magnitude of the constraint stress which is not large enough to affect the porosity evolution. These results are consistent with the observations reported by Frandsen et al. ${ }^{(16)}$. However, there is a significant retardation of porosity evolution in the thin CGO_D layer due to the constraint from the highly shrinking thick CGO_P layer as observed in the final hours of the sintering cycle.

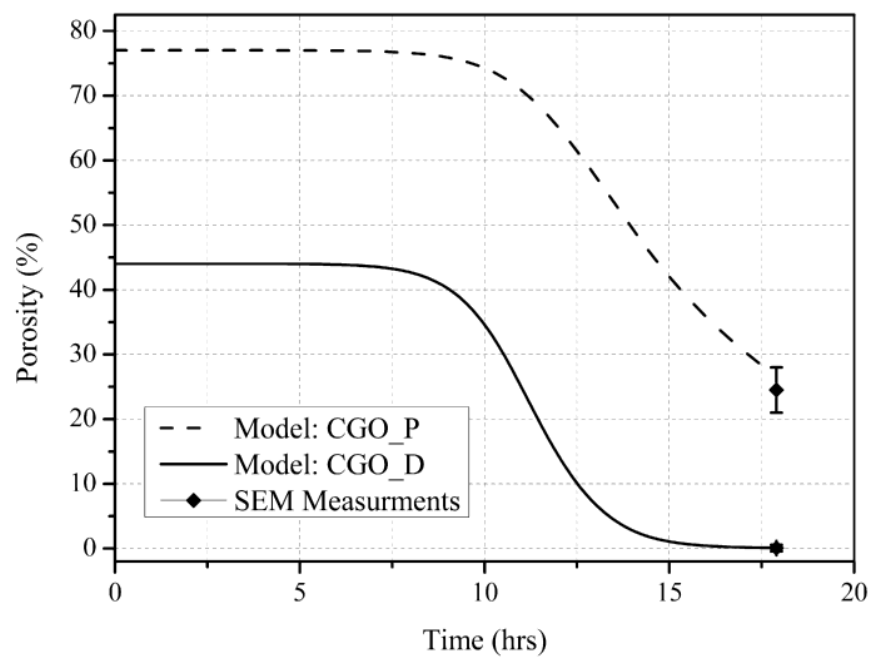

Figure 7: Model predictions for porosity evolutions throughout the sintering process and experimental measurements at the end of the sintering. 


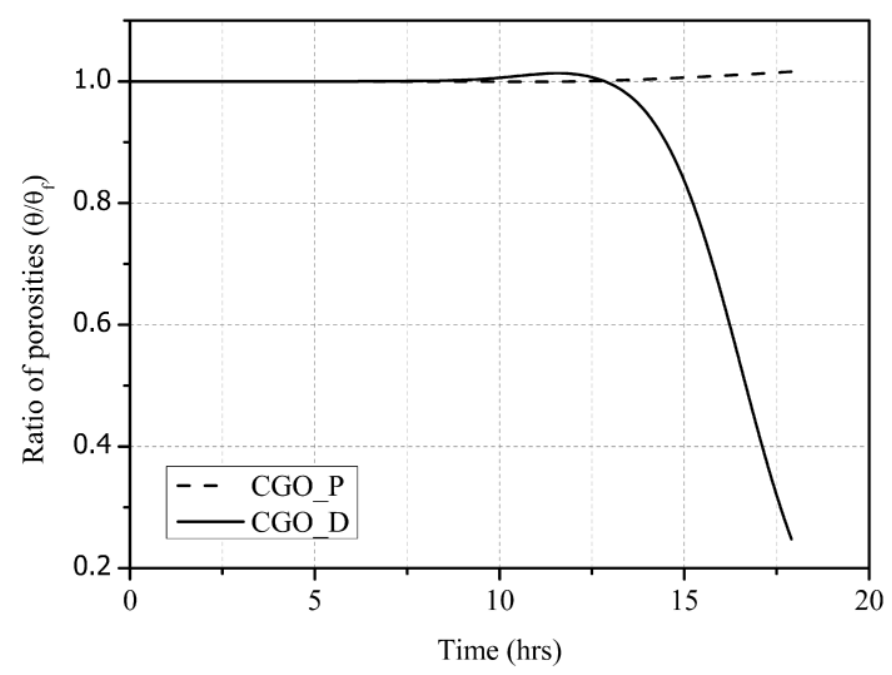

Figure 8: Ratio of porosities in the constrained and free samples during the entire sintering cycle

The large reduction of thickness for CGO_P layer and almost no reduction in thickness for the CGO_D layer are also predicted by the model. The final thickness results predicted by the model agree with the experimental data as shown in Figure 9. In the case of CGO_D a reduction of the thickness followed by thickening of the layer is predicted by the model in the final stage of the sintering. The thickening of the layer is found to be difficult to verify using the SEM images due to the small thickness changes (in order of $1 \mu \mathrm{m})$. However, this can be because of the compressive stress on the already dense CGO_D layer due to the bending moment induced by the weight of the sample in the final stage of the sintering. 


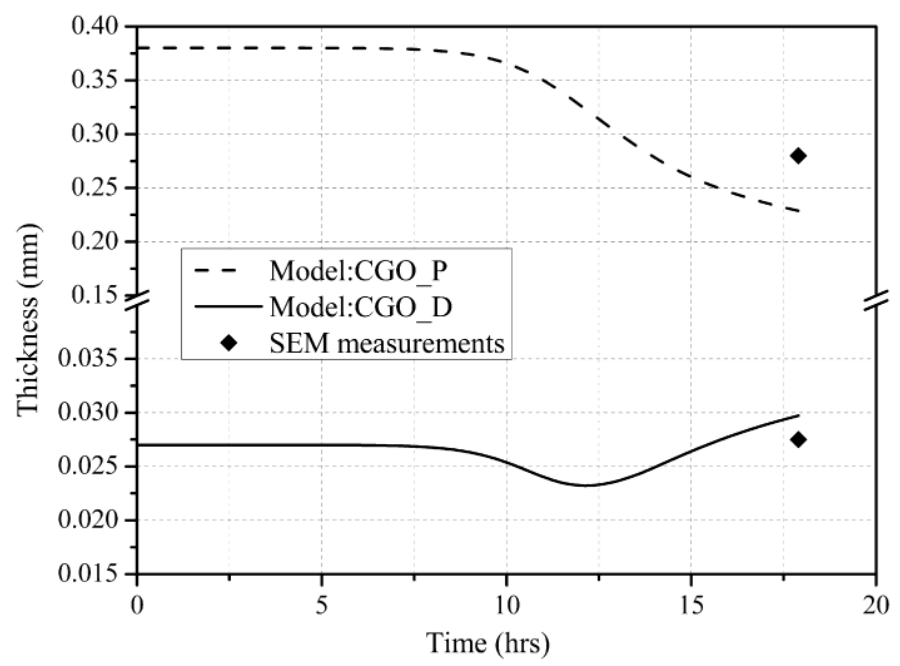

Figure 9: Model predictions for thickness evolutions throughout the sintering process and experimental measurements at the end of the sintering.

\section{Conclusions}

Experimentally observed behaviors of shrinkage and bow development during sintering of bi-layer sample of porous and dense cerium gadolinium oxide (CGO) layers were studied. With the help of the new method proposed, data collected from one optical dilatometry experiment conducted simultaneously for individual layers and asymmetric bi-layer porous structure was sufficient to model kinetics of densification and shape distortion.

The viscous parameters, i.e. the pre-exponential factors and the apparent activation energies in the Arrhenius-type viscosity function were first determined as those providing the least deviation between the free shrinkage strains recorded in the experiment and those obtained by the model.

During the experiment, the bi-layer system is observed to have a reversal of the shape around half of the sintering time and reduction in the bow mainly in the isothermal sintering. The first one is believed to be because of an initial faster shrinkage in the dense layer due to the various additives. The reason for the later reduction in the camber growth was deducted to be due to weight of the sample or gravity, because 
the free sintering samples are observed to sinter throughout the experiment. However the influence of gravity is shown to be minimal in the iso-rate sintering stage as there are high amount of sintering activity in the porous thick layer.

The model is able to capture all the important phenomenon of shape changes observed during the experiment. The significant evolution of layer thickness in the porous thick layer could also justify the need to consider it in the modeling of curvature together with the corresponding linear distribution of strain across the thickness.

\section{Acknowledgments}

The authors would like to acknowledge the support of the Danish Council for Independent Research Technology and Production Sciences (FTP) which is part of The Danish Agency for Science, Technology and Innovation (FI) (Project \# 09-072888) and the US National Aeronautics and Space Administration, Materials Science Program (NNX10AV38G). 


\section{References}

1. Kanters J., Eisele U., Rodel J. Cosintering simulation and experimentation: Case study of nanocrystalline zirconia. J. Am. Ceram. Soc., 2001, 84, 2757-63.

2. Muecke R., Menzler H., Buchkremer P., Stoever D. Cofiring of Thin Zirconia Films During SOFC Manufacturing. J. Am. Ceram. Soc., 2009, 92, S95-S102.

3. Cai Z., Green J., Messing L. Constrained densification of alumina/zirconia hybrid laminates .1. Experimental observations of processing defects. J. Am. Ceram. Soc., 1997, 80, 1929-39.

4. Chang J., Guillon O., Roedel J., Kang S. L. Characterization of warpage behaviour of Gd-doped ceria/NiO-yttria stabilized zirconia bi-layer samples for solid oxide fuel cell application. J. Power Sources, 2008, 185, 759-64.

5. Chiang M., Jean J., Lin S. Effects of green density difference on camber development during the cofiring of a bi-layer glass-based dielectric laminate. Mater. Chem. Phys., 2011, 128, 413-17.

6. Green D., Guillon O., Roedel I. Constrained sintering: A delicate balance of scales. J. Eur. Ceram. Soc., 2008, 28, 1451-66.

7. Lu G., Sutterlin R., Gupta T. Effect of Mismatched Sintering Kinetics on Camber in a LowTemperature Cofired Ceramic Package. J. Am. Ceram. Soc., 1993, 76, 1907-14.

8. Ollagnier J., Guillon O., Roedel J. Constrained Sintering of a Glass Ceramic Composite: I. Asymmetric Laminate. J. Am. Ceram. Soc., 2010, 93, 74-81.

9. Ravi D., Green D. Sintering stresses and distortion produced by density differences in bi-layer structures. J. Eur. Ceram. Soc., 2006, 26, 17-25.

10. Schoenberg E., Green J., Segall E., Messing L., Grader S., Halleck M. Stresses and distortion due to green density gradients during densification. J. Am. Ceram. Soc., 2006, 89, 3027-33.

11. Tzeng S., Jean. J. Stress development during constrained sintering of alumina/glass/alumina sandwich structure. J. Am. Ceram. Soc., 2002, 85, 335-40.

12. Olevsky E. Theory of sintering: from discrete to continuum. Mater. Sc. \& Eng. R-Reports, 1998, 23, 41-100.

13. Olevsky E., Tikare V., Garino T. Multi-scale study of sintering: A review. J. Am. Ceram. Soc., 2006, 89, 1914-22.

14. Lu J., Hng H., Song X., Zhang T., Ma J. Cosintering of a Bimodal Pore Distribution Layered Structure: Constitutive Models and Experiments. J. Am. Ceram. Soc., 2011, 94, 1528-35.

15. Cai P., Green D., Messing G. Constrained densification of alumina/zirconia hybrid laminates .2. Viscoelastic stress computation. J. Am. Ceram. Soc., 1997, 80, 1940-48. 
16. Frandsen H., Olevsky E., Molla T., Esposito V., Bjørk R., Pryds N. Modeling sintering of multi-layers under the influence of gravity, J. Am. Ceram. Soc., 2012, (in press)

17. Cologna M., Sglavo V. Vertical sintering to measure the uniaxial viscosity of thin ceramic layers. Acta Materialia, 2010, 58, 5558-64.

18. Atkinson A., Kim J., Rudkin R., Taub S., Wang X. Stress Induced by Constrained Sintering of 3 YSZ Films Measured by Substrate Creep. J. Am. Ceram. Soc., 2011, 94, 717-24.

19. Lee S., Messing G. \& Green D. Bending creep test to measure the viscosity of porous materials during sintering. J. Am. Ceram. Soc., 2003, 86, 877-82.

20. Reiterer M., Ewsuk K., Arguello J. An Arrhenius-type viscosity function to model sintering using the skorohod-olevsky viscous sintering model within a finite-element code. J. Am. Ceram. Soc., 2006, 89, 1930-35.

21. Raether F., Horn P. Investigation of sintering mechanisms of alumina using kinetic field and master sintering diagrams. J. Eur. Ceram. Soc., 2009, 29, 2225-34.

22. Garino T., Bowen H. Kinetics of Constrained-Film Sintering. J. Am. Ceram. Soc., 1990, 73, 251-57.

23. Scherer G., Brinker C., Roth E. Sol-Gel-Glass: Viscous Sintering. J. Non Cryst. Solids, 1985, 72, 36989.

24. De N., Olevsky E., Esposito V., Molla T., Bjørk R., Pryds N. Sintering of bi-layered porous structures: part II experiments and model applications, J. Am. Ceram. Soc., 2012 (submitted)

25. Tschoegl W., Knauss G., Emeri I. Poisson's ratio in linear viscoelasticity-A critical review. Mech. Time-Depend. Mater., 2002, 6, 3-51.

26. Hsu R., Jean J. Key factors controlling camber behavior during the cofiring of bi-layer ceramic dielectric laminates. J. Am. Ceram. Soc., 2005, 88, 2429-34.

27. De N., Esposito V., Schmidt C., Ramousse S., Pryds N. Densification kinetics analysis of CGO and LSM/CGO tape casting layers.(to be submitted)

28. Rahaman M., Sintering of ceramics, 1st ed., CRC press: Taylor \& Francis Group, 2008 